CrossMark \& click for updates

Cite this: Phys. Chem. Chem. Phys., 2015, 17, 11144

Received 13th February 2015

Accepted 30th March 2015

DOI: $10.1039 / c 5 c p 00935 a$

www.rsc.org/pccp

\section{A high saturation factor in Overhauser DNP with nitroxide derivatives: the role of ${ }^{14} \mathrm{~N}$ nuclear spin relaxation $\dagger$}

\author{
Nikolay Enkin, ${ }^{a}$ Guoquan Liu, ${ }^{\text {a }}$ Maria del Carmen Gimenez-Lopez, ${ }^{\text {b }}$ \\ Kyriakos Porfyrakis, ${ }^{c}$ Igor Tkach ${ }^{a}$ and Marina Bennati*ad
}

\begin{abstract}
Overhauser DNP enhancements of toluene were measured at a magnetic field of 0.35 Tesla in a series of chemically functionalized nitroxide radicals. We observe that the enhancements increase systematically with polarizer size and rotational correlation time. Examination of the saturation factor of ${ }^{14} \mathrm{~N}$ nitroxides by pulsed ELDOR spectroscopy led to a quantitative interpretation of the enhancements, for which the saturation factor increases up to almost unity due to enhanced nuclear $\left({ }^{14} \mathrm{~N}\right)$ relaxation in the nitroxide radical. The observation has a direct impact on the choice of optimum DNP polarizers in liquids.
\end{abstract}

Nuclear magnetic resonance and imaging (NMR/MRI) are versatile techniques for the non-destructive analysis of molecules and biosystems. The sensitivity of these techniques depends on the NMR absorption intensity, which is proportional to the nuclear spin polarization. At room temperature and currently available NMR magnetic fields this quantity amounts to a fraction of a percent. Dynamic nuclear polarization (DNP) provides a means to enhance this polarization and to improve the performance of NMR based techniques. This method relies on polarization transfer from the electron spins to the surrounding nuclei and can enhance the nuclear spin polarization by orders of magnitude..$^{1-4}$

In liquids, DNP is governed by the Overhauser mechanism, ${ }^{5,6}$ which describes the signal enhancement $\varepsilon$ as:

$$
\varepsilon=1-s \cdot f \cdot \xi \cdot\left|\gamma_{\mathrm{e}}\right| / \gamma_{\mathrm{I}}
$$

\footnotetext{
${ }^{a}$ RG EPR spectroscopy, Max-Planck Institute for Biophysical Chemistry, Am Fassberg 11, 37077 Göttingen, Germany

${ }^{b}$ School of Chemistry, University of Nottingham, Nottingham NG7 2RD, UK

${ }^{c}$ Department of Materials, University of Oxford, Oxford OX1 $3 P H$, UK

${ }^{d}$ Department of Chemistry, University of Göttingen, Tammanstr. 2, 37077

Göttingen, Germany. E-mail: guoquan.liu@mpibpc.mpg.de,

marina.bennati@mpibpc.mpg.de

$\dagger$ Electronic supplementary information (ESI) available: Synthesis of FN derivatives, the experimental setup for DNP, CW EPR spectra and power dependence of the central line, ELDOR saturation spectra and time evolution PR-EPR, PR-ELDOR curves for all samples, the relaxation model of the ${ }^{14} \mathrm{~N}$ nitroxide radical, comparison of the experimental and calculated saturation factors, and dependence of ${ }^{14} \mathrm{~N}$ nuclear relaxations on correlation time. See DOI: 10.1039/c5cp00935a
}

where, $\gamma_{\mathrm{e}}$ and $\gamma_{\mathrm{I}}$ are the electron and nuclear gyromagnetic ratios, and $s, f$, and $\xi$ are the saturation, leakage and coupling factors, respectively. ${ }^{6,7}$ The Overhauser parameters $(s, f, \xi)$ depend on the polarizer-solvent system and experimental conditions, such as the magnetic field, microwave (MW) excitation efficiency and sample temperature. ${ }^{8-17}$ If DNP is performed with electron spins in Boltzmann equilibrium, the parameters $s$ and $f$ can achieve values between 0 and 1 whereas $\xi$ has a maximal value of +0.5 for pure dipolar and -1 for pure scalar relaxations, which affords a maximum enhancement of 660. Nevertheless, DNP enhancements might be substantially improved if the electron spins, which transfer their polarization to the nuclei, are far from thermal equilibrium, i.e. in polarized triplet states via optical excitation. ${ }^{18,19}$ Nitroxide radicals can also be polarized optically in solution when they are covalently linked to dyes, such as fullerenes ${ }^{20,21}$ or ferrocenes. ${ }^{22}$ However, in the context of DNP in liquids it is not clear whether a functionalization of the commonly employed nitroxide polarizers, e.g. TEMPOL $(2,2,6,6-$ tetramethylpiperidine 1-oxyl), might attenuate or even destroy their large DNP efficiency. To explore this new avenue, we have synthesized a series of nitroxide derivatives functionalized with fullerene $\mathrm{C}_{60}$ (Fig. 1 and Fig. SI1, ESI $\dagger$ ). Additional introduction of side chains into the fullerene cage increases the molecular size and the solubility in water (after removal of the protecting groups - tBOC). ${ }^{23}$ Despite the solubility of $\mathbf{F N}-2 \mathbf{a}$ and $\mathbf{F N}-\mathbf{3 a}$ in water, the complete series of samples is soluble only in non-polar solvents like toluene, which in turn for DNP have the advantage of low dielectric losses. The EPR lines of the compounds (Fig. 1) in toluene display three well-resolved nitroxide hyperfine (hf) lines (Fig. S2, ESI $\dagger$ ) consistent with spectra in the fast motion regime. We have recently reported that coupling factors of TEMPOL (TL) with ${ }^{1} \mathrm{H}$ in toluene and water are very similar and both controlled by solvent diffusion. ${ }^{24}$ Thus, for all these combined reasons, we employ toluene as a model solvent system for the current DNP investigation. Here, we report the low-field $(0.35 \mathrm{~T} / 9.7 \mathrm{GHz}) \mathrm{DNP}$ performance of these fullerene-nitroxide derivatives. We have observed an unexpected increase of the DNP efficiency with the polarizer size, which led us to investigate the effective 


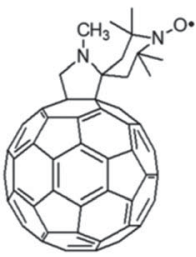

FN

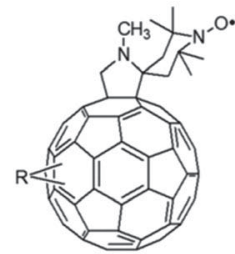

FN-1a

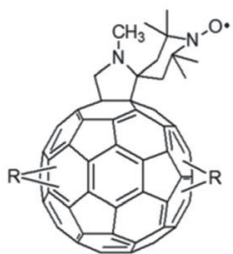

FN-2a

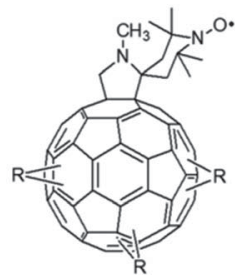

FN-3a

$\mathrm{R}=\mathrm{COBt}-\mathrm{HN} \sim \mathrm{O}_{\mathrm{O}} \sim \mathrm{N}_{\mathrm{H}}^{\mathrm{H}-\mathrm{tBOC}}$

Fig. 1 Fullerene-nitroxide derivatives investigated in DNP experiments (red cycle: link position).

saturation factor using pulsed electron-electron double resonance (ELDOR).

${ }^{1} \mathrm{H}$-NMR toluene spectra were measured at $0.35 \mathrm{~T}$ in toluene solution doped with $\sim 1.5 \mathrm{mM}$ polarizer, which is close to the maximal concentration achievable for compound FN. After microwave irradiation in resonance with the central hf line of the ${ }^{14} \mathrm{~N}$ nitroxide, FN-2a displays a DNP enhancement of -110 and -87 for the ring and methyl protons, respectively (Fig. 2a). Details of the DNP experimental setup and evaluation of the enhancements were reported previously and are summarized in the $\mathrm{ESI} \dagger$ (SI2 and SI3). ${ }^{10,24}$ The negative sign of the enhancement indicates the dominance of dipolar relaxation in the polarization transfer mechanism. This is also valid for all other polarizers studied in this work. The DNP enhancements for the series of polarizers in toluene at the same concentration are presented in Fig. $2 \mathrm{~b}$ for comparison. The enhancement increases from TL to FN-2a and then drops slightly in FN-3a. Because the molecular weight increases from TEMPOL to FN-3a, the result suggests a favorable role of the polarizer size in the observed DNP behavior. However, presuming a DNP mechanism controlled by translational diffusion, ${ }^{25}$ which is yet to be demonstrated, it is not expected that the coupling factors increase with molecular weight.

Therefore, to examine how the Overhauser parameters in eqn (1) are affected by the size of the polarizer, we have measured the saturation factors using the pulsed ELDOR approach for a ${ }^{14} \mathrm{~N}$-nitroxide polarizer with three $\mathrm{hf}$ transitions. In this experiment, the EPR free induction decay (FID) of one hf line is recorded as a function of the microwave frequency of a pumping pulse (Fig. 3a inset). ${ }^{26} \mathrm{~A}$ reduction of the FID signal occurs when the pumping pulse is resonant with one of the three $\mathrm{hf}$ transitions. The largest signal reduction always appears when the pumping frequency coincides with the detection frequency. The other signal reductions reflect saturation transfer from a pumped hf line to the observed one.

The saturation factor $s$ when pumping on one fixed $\mathrm{hf}$ transition is given by the average of the saturation factors of the individual lines $\left(s_{1}, s_{2}, s_{3}\right.$ are for the low field, central field and high field hf lines, respectively):

$$
s=\frac{s_{1}+s_{2}+s_{3}}{3}
$$

In contrast to the two-line case of ${ }^{15} \mathrm{~N}$-nitroxide radicals, ${ }^{26} s_{1}$, $s_{2}$ and $s_{3}$ in ${ }^{14} \mathrm{~N}$-nitroxide radicals has to be determined from

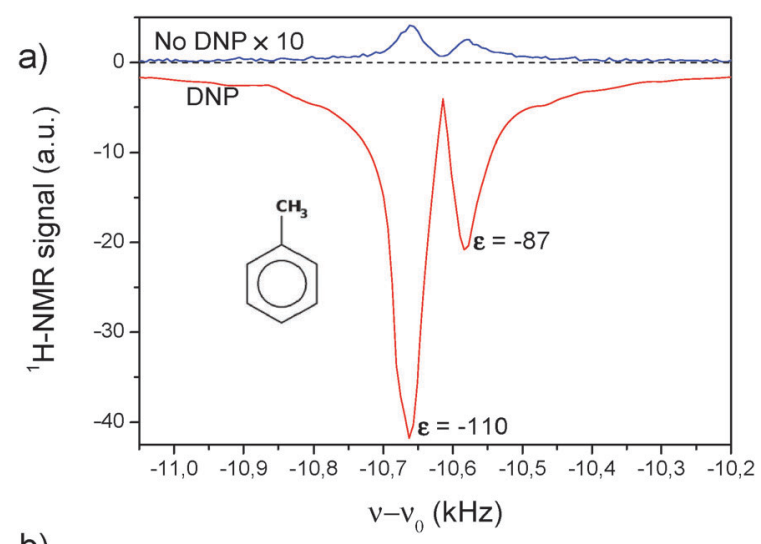

b)

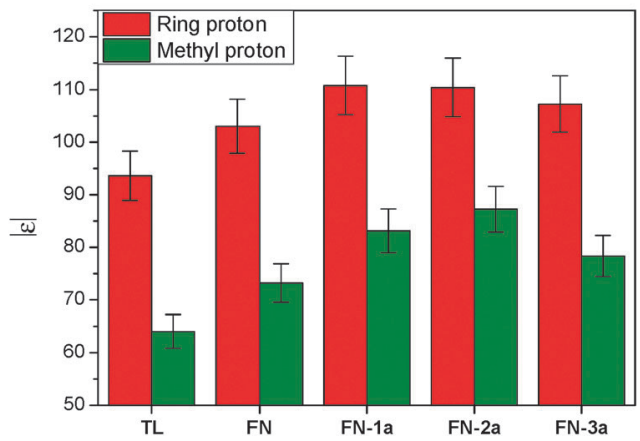

Fig. 2 (a) Boltzmann (blue) and DNP (red) $14 \mathrm{MHz}{ }^{1} \mathrm{H}-\mathrm{NMR}$ spectra recorded in toluene solution of $\mathbf{F N - 2 a}$. (b) DNP enhancements for both ring and methyl protons of toluene using different polarizers. Experimental details: samples were degassed by three freeze-pump-thaw cycles, c $\sim 1.5 \mathrm{mM}$, volume $\sim 20 \mu \mathrm{L}, 1$ or 8 scans for DNP and 128 scans for Boltzmann measurements. MW irradiation was set on the central hf line, $P_{\mathrm{mw}} \approx 3 \mathrm{~W}, t_{\mathrm{irrad}}=20 \mathrm{~s}$. Errors are about $\pm 5 \%$, as also previously reported. ${ }^{24}$

three ELDOR experiments, in which the detection is set once at the time on each hf line consecutively.

To illustrate this procedure, we display the ELDOR experiments for the TL and the FN-2a samples in Fig. 3. In all cases, we observe a complete suppression of the FID when the pumping and detection frequencies coincide. Under such a condition the saturation transfer to the neighboring hf lines reaches the maximum and the obtained saturation factor from the ELDOR experiments corresponds to the maximally achievable $s$ in DNP experiments $\left(s_{\max }\right)$ when pumping one single hf line. ${ }^{26-28}$ For instance, when pumping on the central hf line, 
a)

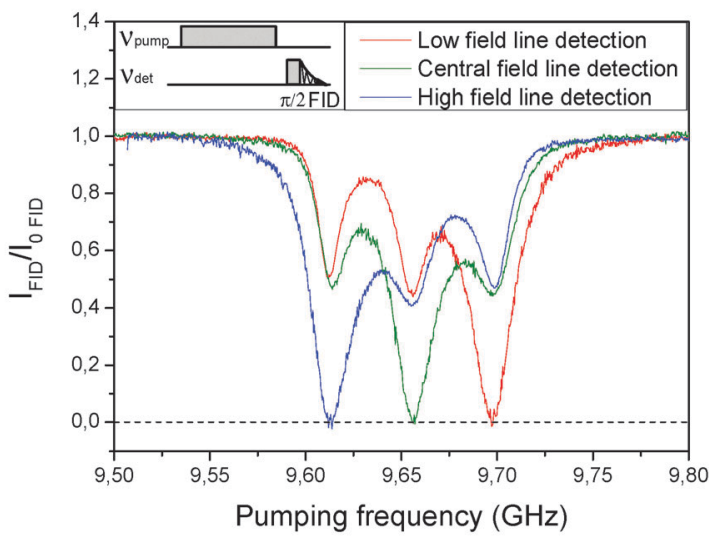

b)

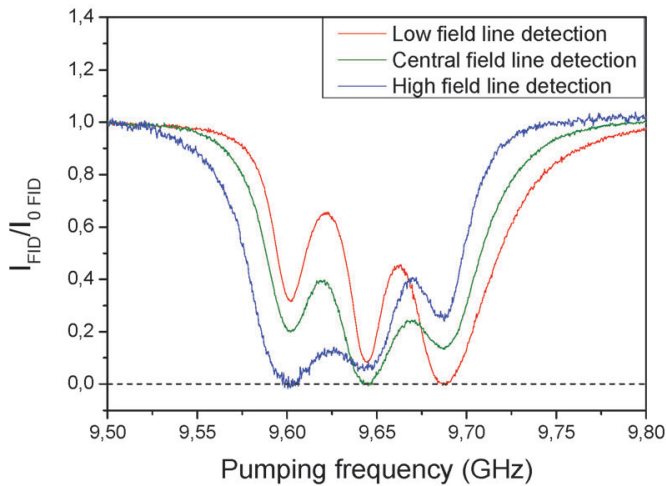

Fig. 3 Normalized FID intensity of TL (a) and FN-2a (b) as a function of the pumping ELDOR frequency. Experimental conditions: $c \sim 1.5 \mathrm{mM}$, $t_{\pi / 2}=24 \mathrm{~ns} ; t_{\text {pump }}=1 \mu \mathrm{s} ; B_{1} \sim 2-3 \mathrm{G}$. The length of the pump pulse was adjusted to observe a complete saturation of the pumped line and to reach the steady state. Inset: pulse sequence in the ELDOR saturation experiment.

$s_{2}$ reaches 1 (green curves in Fig. 3, central peak) and the corresponding $s_{1}$ and $s_{3}$ are given by the reductions of the central peaks in the red and blue curves, respectively. The $s_{\max }$ for pumping on the central hf line could then be calculated according to eqn (2). Additionally, the $s_{\max }$ for pumping on either one of the other two hf lines could be obtained in a similar way. While for $\mathbf{T L}$ (Fig. 3a) the saturation factor appears to be not dependent on the choice of the pumped hf line, for the FN-2a sample it does and the largest saturation factor is observed for pumping on the central hf line, indicating that this is the optimal setup for DNP.

To relate the observed $s$ from the ELDOR to the $s$ in the DNP experiment, the pumped line in DNP must also be completely saturated, as in the ELDOR experiments. An independent measurement of the power dependence of the central hf line (Fig. S3, ESI $\dagger$ ) showed that at the microwave power used in DNP (CW irradiation with $P_{\mathrm{mw}} \approx 3 \mathrm{~W}$ ) this line is indeed completely saturated in all samples $\left(s_{2} \approx 1\right)$. Therefore, in our DNP experiments, the saturation transfer is maximal and $s$ equals that extracted from the ELDOR experiments. The obtained values of $s$ when pumping on the central hf line for all samples are listed in Table 1 (ELDOR spectra for other samples are shown in Fig. S4, ESI $\dagger$ ). We found that $s$ increases with the polarizer size, with a value of 0.71 for TL up to 0.95 for FN-2a and FN-3a. Such high saturation values are very unusual at these low polarizer concentrations ( $\sim 1.5 \mathrm{mM})$. For comparison, a similar saturation value can be achieved with TEMPO- ${ }^{2} \mathrm{H}-{ }^{15} \mathrm{~N}$ only at $\geq 5 \mathrm{mM}$ concentration in toluene or $\geq 25 \mathrm{mM}$ in water. $^{24,26}$

In order to investigate the origin of the increased saturation factors, we analyzed the population dynamics during saturation transfer by recording polarization recovery EPR (PR-EPR) and ELDOR (PR-ELDOR) curves for all samples in toluene (Fig. 4 and Fig. S5, ESI $\dagger$ ). The pulse sequence for both types of experiments was described earlier ${ }^{24,26,29}$ and is shown in the inset of Fig. 4. For the polarization recovery process in the ${ }^{14} \mathrm{~N}$-nitroxide radical, the evolution of the detected FID signals $i_{1,2,3}$ (index denotes the hf line) after a pumping pulse can be described within a model

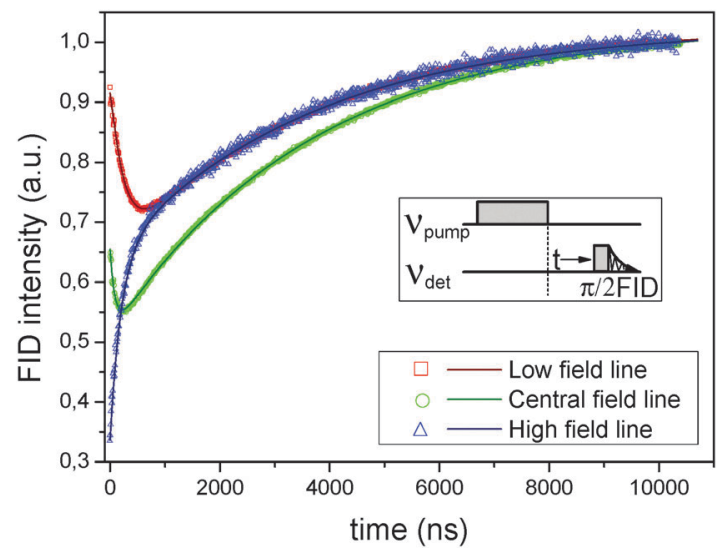

Fig. 4 Polarization recovery (PR) curves and fits (solid lines) of the FN-2a FID for pumping on the high field line and detect at the same line (PR-EPR, blue triangles) or on one of the other hf lines (PR-ELDOR, green circles and red squares). Exp. conditions: $c \sim 1.5 \mathrm{mM}, t_{\pi / 2}=24 \mathrm{~ns} ; t_{\text {pump }}=0.1 \mu \mathrm{s}$; $B_{1} \sim 2-3 G$. The length of the pump pulse is shorter here as the steady state is not required. Inset: pulse sequence for PR-ELDOR and PR-EPR.

Table 1 Summary of DNP parameters, relaxation times and Heisenberg exchange rate constants for toluene solutions of TL and FN derivatives. In DNP, microwave irradiation was set on the central hf line. Errors in $T_{1 \mathrm{e}}, T_{1 \mathrm{n}}$, and $K_{\mathrm{x}}$ from fits of PR data as well as $\tau_{\mathrm{c}}$ are estimated up to $20 \%$. Errors in $\varepsilon$ (from DNP) are about $5 \%$, whereas the errors in $f$ and $s$ are $\leq 5 \%$. The resulting error in $\xi$ is about $\pm 10 \%$. However, errors in the trends among the samples are much less, as pointed out previously ${ }^{24}$

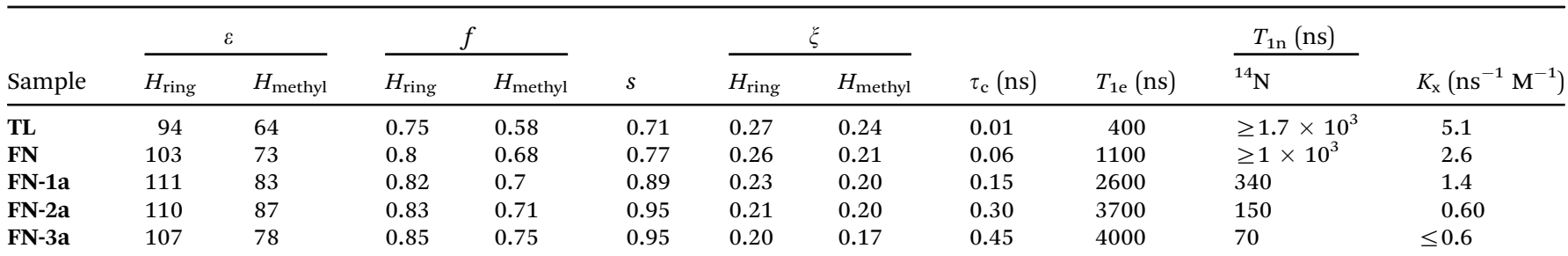


that takes into account the electron and ${ }^{14} \mathrm{~N}$ nuclear-spin lattice relaxation as well as Heisenberg exchange. This model is discussed in detail in SI4 (ESI $\dagger$ ) and leads to a triple exponential behaviour ${ }^{29}$ $(i=1,2,3)$ of the $\mathrm{hf}$ lines:

$$
I_{i}=A_{i} \mathrm{e}^{-2 w_{\mathrm{e}} t}+B_{i} \mathrm{e}^{-\left(2 w_{\mathrm{e}}+3 w_{\mathrm{n}}+K_{\mathrm{x}} N\right) t}+C_{i} \mathrm{e}^{-\left(2 w_{\mathrm{e}}+w_{\mathrm{n}}+K_{\mathrm{x}} N\right) t}
$$

where $2 w_{\mathrm{e}}$ and $2 w_{\mathrm{n}}$ are the electron and nuclear spin-lattice relaxation rates $\left(1 / T_{1 \mathrm{e}}=2 w_{\mathrm{e}}, 1 / T_{1 \mathrm{n}}=2 w_{\mathrm{n}}\right)$, respectively, $K_{\mathrm{x}}$ is the normalized Heisenberg spin exchange rate per mole and $N$ is the polarizer concentration. The detailed derivation of this equation is presented in SI4 (ESI $\dagger$ ). The amplitudes (A, B, C) are given by the initial conditions and eigenvectors. Particularly, when pumping on the central hf line the triple exponential model is reduced to a bi-exponential one with the last term in eqn (3) vanished (eqn (S14) in the ESI $\dagger$ ). Therefore, to determine all three rates $2 w_{\mathrm{e}}, 2 w_{\mathrm{n}}$ and $K_{\mathrm{x}} N$, experiments with pumping either on the high or low field hf lines are required. Representative data for pumping on the high field line are displayed in Fig. 4 (data for all other samples are shown in Fig. S5, ESI $\dagger$ ). Fitting of three recovery curves with shared exponentials delivers values for $2 w_{\mathrm{e}}, 2 w_{\mathrm{n}}$ and $K_{\mathrm{x}} N$ rates. The values of $2 w_{\mathrm{e}}$ and $2 w_{\mathrm{n}}$, expressed as $T_{1 \mathrm{e}}$ and $T_{1 \mathrm{n}}$, as well as $K_{\mathrm{x}}$ for all samples are listed in Table 1.

The results show that the electron spin-lattice relaxation rate $2 w_{\mathrm{e}}$ and the Heisenberg exchange rate $K_{\mathrm{x}}$ decrease with the size of the nitroxide derivative, while the ${ }^{14} \mathrm{~N}$ nuclear relaxation rate $\left(2 w_{\mathrm{n}}\right)$ increases. After determination of the rotational correlation time of each sample from the CW EPR spectra (Fig. S2 (ESI $\dagger$ ) and Table 1), we plot the rates $2 w_{\mathrm{n}}$ and $K_{\mathrm{x}} N$ against the rotational correlation time in Fig. 5 . The ${ }^{14} \mathrm{~N}$ nuclear relaxation increases with the correlation time and crosses over the Heisenberg exchange rate (for $1.5 \mathrm{mM}$ polarizer) at a correlation time of $\sim 0.15 \mathrm{~ns}$. Such an increase in the nuclear relaxation rate finds some precedents in ELDOR studies of the ${ }^{15} \mathrm{~N}$-TEMPO radical in solvents of different viscosities ${ }^{30}$ as well as studies of spin labeled lipids. ${ }^{29}$ In solutions of nitroxide

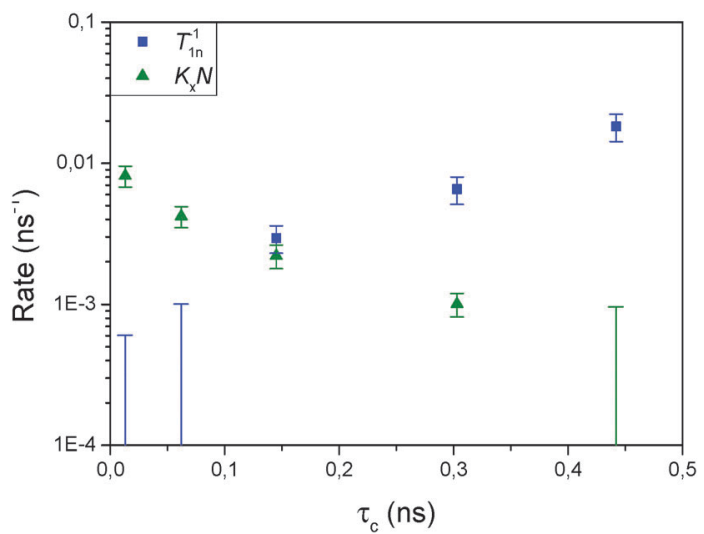

Fig. $5{ }^{14} \mathrm{~N} T_{1 \mathrm{n}}{ }^{-1}\left(2 w_{\mathrm{n}}\right)$ and $K_{\mathrm{x}} N(\mathrm{~N} \equiv \mathrm{c} \approx 1.5 \mathrm{mM})$ from the polarization recovery spectra, as a function of the rotational correlation time of the nitroxide derivatives. Values of $T_{1 \mathrm{n}}{ }^{-1}$ for TL and FN and the value of $K_{\mathrm{x}} N$ for FN-3a turned out to be negligible in the fits and are given as upper limits. An error of up to $20 \%$ was estimated by repeated experiments at various concentrations. radicals, the ${ }^{14} \mathrm{~N}$ nuclear relaxation in this dynamic range (ps-ns) was proposed to be governed by the electron-nuclear dipolar mechanism. ${ }^{30}$ This has contributions from two spectral densities centered at the electron Larmor $\omega_{\mathrm{e}}$ and at the nuclear resonance frequencies $\omega_{\mathrm{n}}$, the latter dominated by the hyperfine coupling: ${ }^{30,31}$

$$
\frac{1}{T_{1 \mathrm{n}}}=k \cdot\left(\frac{7 \cdot \tau_{\mathrm{c}}}{1+\omega_{\mathrm{e}}{ }^{2} \tau_{\mathrm{c}}{ }^{2}}+\frac{3 \cdot \tau_{\mathrm{c}}}{1+\omega_{\mathrm{n}}{ }^{2} \tau_{\mathrm{c}}{ }^{2}}\right)
$$

The rotational correlation time $\tau_{\mathrm{c}}$ of all our samples in toluene falls in a regime of $\leq 1 \mathrm{~ns}$, for which the spectral density $J\left(\omega_{\mathrm{n}}\right)$ increases with the rotational correlation time, as displayed in a simulation in Fig. S6 (ESI $\dagger$ ).

To elucidate the direct role of ${ }^{14} \mathrm{~N}$ nuclear-spin lattice relaxation in the saturation factor, we have derived analytical solutions for the saturation transfer (ELDOR) between hf lines (SI4, ESI $\dagger$ ). When saturating the central hf line $\left(s_{2}=1\right)$, the maximal saturation factors $s_{1}$ and $s_{3}$ of the other hf lines are given by:

$$
s_{1}=s_{3}=1-\frac{1}{1+\frac{1}{6 w_{\mathrm{e}}}\left(3 w_{\mathrm{n}}+K_{\mathrm{x}} N\right)}
$$

These expressions appear to be mostly consistent with those given in ref. 32 for the $I=1$ system; however, the definition of some factors in ref. 32 is not entirely clear. While the Heisenberg exchange rate decreases with the molecular size (Fig. 5), because it is proportional to the diffusion constant, ${ }^{32-34}$ eqn (5) shows that the nuclear relaxation rate $w_{\mathrm{n}}$ combined with $w_{\mathrm{e}}$ is responsible for the observed increase of the saturation factors in our samples. To check for consistency between the theory (eqn (5)) and the ELDOR data, we calculated the maximal $s_{1}$ and $s_{3}$ according to eqn (5) and with values listed in Table 1 . The obtained values (Table S2, ESI $\dagger$ ) show good agreement with the ELDOR experiments within uncertainties of 10 to $15 \%$.

Moreover nuclear relaxation and Heisenberg exchange exhibit different dependences on the polarizer concentration (eqn (5)). We measured $s$ in $\mathbf{T L}$ and $\mathbf{F N}-2 \mathbf{a}$ for a series of concentrations from ELDOR experiments (Fig. 6). As expected, $s$ in TL solutions increases with the polarizer concentration due to the dominant role of Heisenberg exchange. In contrast, $s$ in $\mathbf{F N - 2 a}$ solutions remains nearly constant up to the maximal attainable concentration of $1.6 \mathrm{mM}$. These observations provide direct evidence for the two different mechanisms involved in the saturation behavior of nitroxide EPR lines in this concentration regime.

The present results demonstrate that fast nuclear relaxation can enhance the saturation factor in DNP. This hypothesis was previously raised by Armstrong and Han, ${ }^{17,35}$ and conveyed to tethered nitroxide radicals. Our present study reports the first experimental evidence, bringing an open discussion in the literature to a more consistent picture. Nevertheless our results indicate that the dependence of $s$ on $\tau_{\mathrm{c}}$ is quite dramatic, basically allowing any value between 0.33 (no ELDOR effect) and 1 especially at low concentrations (Fig. 6), and needs to be considered in detail for any new polarizer system.

The advantage of EPR saturation driven by fast nuclear relaxation instead of Heisenberg exchange is its independence 


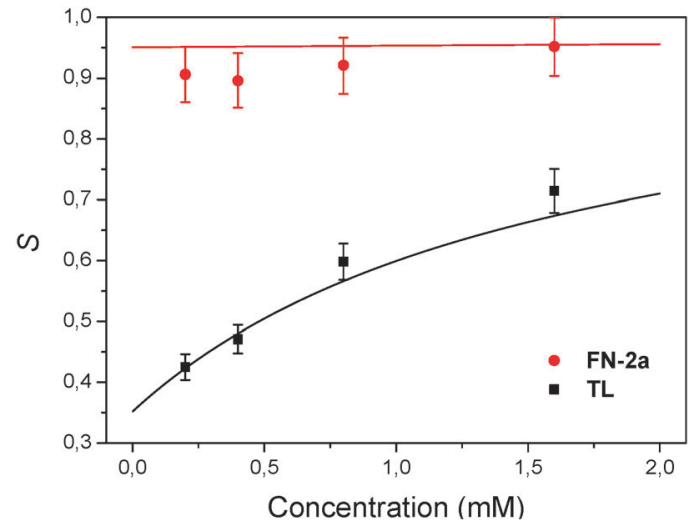

Fig. 6 Saturation factor as a function of the polarizer concentration when saturating the central hf line. Experimental values (red circles and black squares) are determined from pulse ELDOR experiments as described in Fig. 3. Red and black lines are theoretical curves for FN-2a and TL in toluene using eqn (2) and (5) as well as $w_{e}, w_{n}$ and $K_{x} N$ from Table 1. The error in $s$ is basically given by the noise level (base line) in the FID detection of the ELDOR experiments and amounts to about $5 \%$.

of radical concentration. High saturation factors and therefore DNP efficiency can be achieved at a relatively low polarizer concentration $(c \approx 1 \mathrm{mM})$.

To assess the application potential of functionalized nitroxides, we have finally evaluated the leakage factors from the measurements of the ${ }^{1} \mathrm{H}$ nuclear spin-lattice relaxation for ring and methyl protons and subsequently calculated the coupling factor $\xi$ based on eqn (1) (Table 1). There is some but not substantial decrease of the coupling factor $\xi$ between TL and FN-3a $(\Delta \xi \approx 25 \%)$. This behavior is consistent with the current mechanistic model for DNP in nitroxide/toluene, which is controlled by dipolar relaxation driven by molecular diffusion and the accessibility to the nitroxide electron spin center. ${ }^{17,25,36}$ When the molecular size increases, the diffusional correlation time $\tau_{\mathrm{D}}=d^{2} /\left(D_{\mathrm{S}}+D_{\mathrm{P}}\right)$ is expected to increase as well, where $d$ is the distance of closest approach between electron and nuclear spins and $D$ is the diffusion coefficient of the solvent and polarizer, respectively. ${ }^{25}$ An increase of $\tau_{\mathrm{D}}$ finally leads to a decrease in the coupling factor. Nevertheless, in the present case this decrease in $\xi$ is largely compensated by the increase in $s$ and also $f$, leading overall to large enhancements. These findings shed light on the design of new polarizers for DNP in liquids. Work is in progress to examine the saturation behavior at a higher magnetic field (e.g. $3.3 \mathrm{~T}$ ).

\section{Acknowledgements}

We would like to thank Vladimir Belov at the MPIBPC facility for Chemical Synthesis for the synthesis of the FN sample. We acknowledge financial support from the Max Planck Society and the COST Action TD1103 (European Network on Hyperpolarization). NE acknowledges the Graduate School for Neurosciences, Biophysics and Molecular Biosciences in Göttingen (GGNB) for financial support. GL thanks the Alexander von
Humboldt Foundation for a fellowship. KP acknowledges EPSRC for support (EP/K030108/1).

\section{Notes and references}

1 T. Maly, G. T. Debelouchina, V. S. Bajaj, K. N. Hu, C. G. Joo, M. L. Mak-Jurkauskas, J. R. Sirigiri, P. C. A. van der Wel, J. Herzfeld, R. J. Temkin and R. G. Griffin, J. Chem. Phys., 2008, 128, 052211.

2 J. H. Ardenkjaer-Larsen, B. Fridlund, A. Gram, G. Hansson, L. Hansson, M. H. Lerche, R. Servin, M. Thaning and K. Golman, Proc. Natl. Acad. Sci. U. S. A., 2003, 100, 10158-10163.

3 M. Bennati, I. Tkach and M.-T. Türke, in Electron Paramagnetic Resonance, ed. B. C. Gilbert, D. M. Murphy and V. Chechik, RSC Publishing, Cambridge, 2011, vol. 22, pp. 155-182.

4 C. Y. Cheng and S. I. Han, Annu. Rev. Phys. Chem., 2013, 64, 507-532.

5 A. W. Overhauser, Phys. Rev., 1953, 92, 411-415.

6 D. Hausser and D. Stehlik, Adv. Magn. Reson., 1968, 3, 79-139.

7 W. Müller-Warmuth and K. Meise-Gresch, Adv. Magn. Reson., 1983, 11, 1-45.

8 P. Höfer, G. Parigi, C. Luchinat, P. Carl, G. Guthausen, M. Reese, T. Carlomagno, C. Griesinger and M. Bennati, J. Am. Chem. Soc., 2008, 130, 3254-3255.

9 P. Höfer, P. Carl, G. Guthausen, T. Prisner, M. Reese, T. Carlomagno, C. Griesinger and M. Bennati, Appl. Magn. Reson., 2008, 34, 393-398.

10 M. T. Turke, I. Tkach, M. Reese, P. Hofer and M. Bennati, Phys. Chem. Chem. Phys., 2010, 12, 5893-5901.

11 (a) V. Denysenkov, M. J. Prandolini, M. Gafurov, D. Sezer, B. Endeward and T. F. Prisner, Phys. Chem. Chem. Phys., 2010, 12, 5786-5790; (b) J. G. Krummenacker, V. P. Denysenkov, M. Terekhov, L. M. Schreiber and T. F. Prisner, J. Magn. Reson., 2012, 215, 94-99.

12 C. Griesinger, M. Bennati, H. M. Vieth, C. Luchinat, G. Parigi, P. Hofer, F. Engelke, S. J. Glaser, V. Denysenkov and T. F. Prisner, Prog. Nucl. Magn. Reson. Spectrosc., 2012, 64, 4-28.

13 E. V. Kryukov, M. E. Newton, K. J. Pike, D. R. Bolton, R. M. Kowalczyk, A. P. Howes, M. E. Smith and R. Dupree, Phys. Chem. Chem. Phys., 2010, 12, 5757-5765.

14 E. V. Kryukov, K. J. Pike, T. K. Y. Tam, M. E. Newton, M. E. Smith and R. Dupree, Phys. Chem. Chem. Phys., 2011, 13, 4372-4380.

15 G. H. A. van der Heijden, A. P. M. Kentgens and P. J. M. van Bentum, Phys. Chem. Chem. Phys., 2014, 16, 8493-8502.

16 M. Gafurov, V. Denysenkov, M. J. Prandolini and T. F. Prisner, Appl. Magn. Reson., 2012, 43, 119-128.

17 J. M. Franck, A. Pavlova, J. A. Scott and S. Han, Prog. Nucl. Magn. Reson. Spectrosc., 2013, 74, 33-56.

18 A. Henstra, T. S. Lin, J. Schmidt and W. T. Wenckebach, Chem. Phys. Lett., 1990, 165, 6-10.

19 K. Tateishi, M. Negoro, A. Kagawa and M. Kitagawa, Angew. Chem., Int. Ed., 2013, 52, 13307-13310.

20 F. Arena, F. Bullo, F. Conti, C. Corvaja, M. Maggini, M. Prato and G. Scorrano, J. Am. Chem. Soc., 1997, 119, 789-795. 
21 H. Moons, E. Goovaerts, V. P. Gubskaya, I. A. Nuretdinov, C. Corvaja and L. Franco, Phys. Chem. Chem. Phys., 2011, 13, 3942-3951.

22 F. Conti, C. Corvaja, C. Gattazzo, A. Toffoletti, P. Bergo, M. Maggini, G. Scorrano and M. Prato, Phys. Chem. Chem. Phys., 2001, 3, 3526-3531.

23 C. F. Richardson, D. I. Schuster and S. R. Wilson, Org. Lett., 2000, 2, 1011-1014.

24 N. Enkin, G. Q. Liu, I. Tkach and M. Bennati, Phys. Chem. Chem. Phys., 2014, 16, 8795-8800.

25 M. Bennati, C. Luchinat, G. Parigi and M.-T. Türke, Phys. Chem. Chem. Phys., 2010, 12, 5902-5910.

26 M.-T. Turke and M. Bennati, Phys. Chem. Chem. Phys., 2011, 13, 3630-3633.

27 J. H. Freed, J. Chem. Phys., 1965, 43, 2312-2332.
28 J. H. Freed, J. Phys. Chem., 1967, 71, 38-51.

29 (a) J. J. Yin, M. Pasenkiewicz-Gierula and J. S. Hyde, Proc. Natl. Acad. Sci. U. S. A., 1987, 84, 964-968; (b) C. Popp and J. S. Hyde, Proc. Natl. Acad. Sci. U. S. A., 1982, 79, 2559-2563.

30 B. H. Robinson, D. A. Haas and C. Mailer, Science, 1994, 263, 490-493.

31 I. Solomon, Phys. Rev., 1955, 99, 559-565.

32 J. S. Hyde, J. C. W. Chen and J. H. Freed, J. Chem. Phys., 1968, 48, 4211-4226.

33 J. D. Currin, Phys. Rev., 1962, 126, 1995-2001.

34 B. L. Bales and M. Peric, J. Phys. Chem. B, 1997, 101, 8707-8716.

35 B. D. Armstrong and S. Han, J. Chem. Phys., 2007, 127, 104508. 36 D. Sezer, Phys. Chem. Chem. Phys., 2013, 15, 526-540. 